\title{
Criteria for Determining the Need for Admission of People with Dementia to Dementia Nursing Wards of Psychiatric Hospitals in Japan
}

\author{
Tetsuko Takaoka1 ([), Hatsumi Atsuko², Kikuchi Yaeko³ , Ruriko Kidachi' ${ }^{4}$ (]) \\ ${ }^{1}$ Department of Nursing, Faculty of Human Sciences, Hokkaido Bunkyo University, Eniwa, Japan \\ ${ }^{2}$ Graduate School of Nursing and Social Services, Health Sciences University of Hokkaido, Tobetsu, Japan \\ ${ }^{3}$ Ohyachi Hospital, Sapporo, Japan \\ ${ }^{4}$ Hirosaki University Graduate School of Health Sciences, Hirosaki, Japan \\ Email: tetsumo8@do-bunkyodai.ac.jp
}

How to cite this paper: Takaoka, T., Atsuko, H., Yaeko, K. and Kidachi, R. (2021) Criteria for Determining the Need for Admission of People with Dementia to Dementia Nursing Wards of Psychiatric Hospitals in Japan. Open Journal of Nursing, 11, 702-714.

https://doi.org/10.4236/ojn.2021.118060

Received: June 16, 2021

Accepted: August 21, 2021

Published: August 24, 2021

Copyright $\odot 2021$ by author(s) and Scientific Research Publishing Inc. This work is licensed under the Creative Commons Attribution International License (CC BY 4.0).

http://creativecommons.org/licenses/by/4.0/

(c) (i) Open Access

\begin{abstract}
Purpose: This study aims to establish criteria to determine the need for admission of people with dementia to dementia nursing wards of psychiatric hospitals-based on the experience of nurses working in dementia nursing wards. Methods: Semi-structured interviews were conducted with nurses who had worked in dementia nursing wards of psychiatric hospitals for more than 3 years, to collect data related to the "condition at the time of admission and the process of hospitalization of dementia patients". Data were analyzed using the content analysis approach. Focusing on "What is the condition of patients with dementia admitted to the dementia nursing ward?", we created codes according to similarities in the meaning, and classified these into categories where they were evaluated to fully fit in. Results and Discussion: The analysis yielded 4 categories, and 44 codes. The four categories are as follows: [Appearance of a state where self-control is difficult] which expresses a state where behavioral and psychological symptoms of dementia (BPSD) have appeared, and the remaining three categories [Insufficient support provided], [Appearance of symptoms that make living difficult], and [Appearance of physical symptoms that require treatment] express the states that may trigger the appearance of BPSD. These show that the appearance of BPSD is a criterion for determining hospitalization. By providing support to prevent the states described in the 44 codes, nurses may help people with dementia avoid being hospitalized in dementia nursing wards in psychiatric hospitals.
\end{abstract}




\section{Keywords}

Dementia, Dementia Nursing Wards in Psychiatric Hospitals, Criteria in

Determining the Need for Admission

\section{Introduction}

\subsection{Background}

In Japan, the percentage of older people in the population in 1970 was $7.1 \%$, and this number had doubled (14.1\%) in 1994, showing that Japanese society is more rapidly aging than Western European countries [1]. With the increase in the number of older people, the number of people with dementia also increased and had exceeded 5 million in 2018, and it is estimated that one in seven of the older people, aged $>65$, will suffer from dementia [2]. The Cabinet Office of Japan has estimated that $20 \%$ of older people will suffer from dementia in 2025 [3]. With this background, and as the number of people with dementia is expected to increase in proportion to the increase in the number of older people in Japan, it is very important to take appropriate measures. On the assumption that anyone among the older people could develop dementia, the Ministry of Health, Labour and Welfare (MHLW)) has worked to promote measures for addressing dementia with two main objectives, to achieve and enable "coexistence" with working at "prevention" of dementia [2]. The MHLW aims to control the onset of dementia and enable people with dementia to continue their daily lives with hope and dignity even when they have dementia, while placing importance on the opinions of the families of the people with dementia. According to the MHLW, "coexistence" here means working towards a society where people with dementia are able to coexist in the same communities as the rest of the population [2]. This suggests that it is considered desirable for them to be able to continue their daily lives in the communities with people they are familiar with even when they have dementia. However, in Japan there are many people with dementia who are living in long-term nursing facilities including group homes away from such desirable conditions. Further, many patients with prominent behavioral and psychological symptoms of dementia (BPSD) are cared for in dementia nursing wards of psychiatric hospital [4]. Previous studies on dementia nursing at psychiatric hospitals have reported mainly about care responses to severely difficult cases caused by severe BPSD including BPSD conditions [5], and care details and outcomes [6], as well as there are reports focusing on factors related to BPSD after admission, including family conditions [7], BPSD-related factors [8], and the actual conditions of psychiatric hospitals [9]. We have located no studies that report on the factors that exist before the time of admission.

Considering the situation described above, it is considered to be necessary to take early and proactive measures by investigating patient conditions at the time of admission and the factors that led to the admission which would make it possi- 
ble for patients with dementia to be discharged early from dementia nursing wards of psychiatric hospitals. To remedy this, the present study aims to clarify the conditions of patients at the time of admission and the process of hospitalization for the dementia patients through interviews with nurses in dementia nursing wards of the psychiatric hospitals who have experienced a variety of difficult cases. A better understanding of these matters, the states and conditions prior to admission, will make it possible to provide appropriate treatment for people with dementia at an early stage of hospitalization, and to shorten the period when the hospitalized patients with dementia and their family caregivers suffer from the burden of BPSD. Such information would also be useful in establishing criteria for determining standards for the admission to dementia nursing wards of psychiatric hospitals. Further, sharing these results with the local community will lead to prevention of BPSD and make it possible for people with dementia to the continue their daily lives in the area where they are accustomed to living.

\subsection{Purpose}

This study aims to establish criteria in determining the need for admission of people with dementia to the dementia nursing wards of psychiatric hospitals based on the experience of nurses working in dementia nursing wards.

\section{Methods}

\subsection{Study Participants}

We sent letters requesting study cooperation to the directors of nursing departments of psychiatric hospitals that have dementia nursing wards in a government-designated city in Hokkaido, Japan. Of these, we determined the hospitals that expressed consent in the study participation as participating hospitals. The inclusion criteria for participants were nurses who had worked in the dementia nursing wards of psychiatric hospitals for more than 3 years. Benner [10] states that one characteristic of mid-career nurses is that they conduct nursing activities based on the rating of a situation, seeing a situation as a whole rather than from the perspective of a specific aspect they are presented with. Because Benner also defines mid-career nurses as those with three to five years of experience in the same or similar departments [10], we determined nurses with more than three years of nursing experience to be suitable for inclusion in the present study. The research staff at the participating hospitals selected nurses who met this criterion in selecting candidates for participation. We visited the selected nurses and explained the study outlines in person based on previously developed "Study Outline" material for explanation, and included nurses who expressed consent to participation in the study after this explanation.

\subsection{Data Collection}

Data were collected by conducting semi-structured interviews using a question- 
naire. Demographic data were collected from the questionnaire, and in the semistructured interview, we collected data related to the "condition at the time of admission and the process of hospitalization of dementia patients" based on the hospitalization cases the participants (nurses) had actually been involved in. In the interviews we asked about the "purpose of the hospitalization of inpatient cases", the "process up until the hospitalization", and "the reasons why (you thought) it could have been better if the patients had been hospitalized earlier". The length of the interview was about one hour per participant, in consideration of the potential fatigue felt by the participants. All the interviews were recorded on an electric device and the interviewer took notes with the consent of the participants. The place and date of the time of the interview were decided by the preference of the participants.

\subsection{Data Analysis}

Data were analyzed using the content analysis approach by Bernard, et al. [11]. We carefully read the transcriptions from the recorded interviews and divided the data into meaningful contexts (one unit per sentence). Focusing on "statements related to the admission of patients with dementia to the dementia nursing wards", and we decided whether to extract further contexts. Next, focusing on "What is the condition of patients with dementia admitted to the dementia nursing ward?", we created codes according to similarities in the meaning, and classified these into categories when they were evaluated to fully fit in. The researchers further examined the validity of the extracted codes and categories.

\subsection{Ethical Considerations}

The probability of participants experiencing the participation as a physical and psychological imposition in this study was low. However, there was the concern that participants could think that refusing participation would result in some disadvantage. For this reason, we explained and promised that such disadvantage would not happen, that the personal information of the participants would be protected, that participants can discontinue the participation even after they consented to the cooperation, that such withdrawal would not cause any disadvantages, that the reason for the withdrawal would not be queried, and that the data of the participants would be destroyed immediately when we received a notice of withdrawal. This study was approved by an ethical review by the institutions to which the researchers belong (No30010).

\section{Results}

\subsection{Demographics of Participants}

Table 1 shows the demographics of the participants. There were 18 participants: 15 females and 3 males with a mean age of 42.1 years $(S R=8.8)$. The final academic training was vocational schools (12 participants), universities (5), or graduate school (1). The mean length of working as a nurse was 16.1 years $(\mathrm{SD}=9.5)$, the 
Table 1. Demographics of participants $(n=18)$.

\begin{tabular}{|c|c|c|}
\hline & Item & $\mathrm{n}$ \\
\hline \multirow{2}{*}{ Sex } & Female & 15 \\
\hline & Male & 3 \\
\hline \multirow{2}{*}{ Age (years) } & Mean & 42.1 \\
\hline & $\mathrm{SD}$ & 8.8 \\
\hline \multirow{3}{*}{ Final academic background } & Vocational school & 12 \\
\hline & University/college & 5 \\
\hline & Graduate school & 1 \\
\hline \multirow{2}{*}{ Length of working as a nurse (years) } & Mean & 16.1 \\
\hline & SD & 9.5 \\
\hline \multirow{2}{*}{$\begin{array}{l}\text { Length of working in the dementia } \\
\text { nursing wards (years) }\end{array}$} & Mean & 4.4 \\
\hline & $\mathrm{SD}$ & 2.5 \\
\hline \multirow{2}{*}{ Professional certification } & Certified nurses in dementia nursing & 3 \\
\hline & Certified nurse specialist in gerontological nursing & 1 \\
\hline \multirow{6}{*}{$\begin{array}{l}\text { Ward where participants were } \\
\text { previously working }\end{array}$} & Surgery & 3 \\
\hline & Internal medicine & 2 \\
\hline & Surgery and internal mixed & 2 \\
\hline & Operating room & 1 \\
\hline & Psychiatry & 9 \\
\hline & None & 1 \\
\hline \multirow{2}{*}{$\begin{array}{l}\text { Length of working in general wards } \\
\text { (years) }\end{array}$} & Mean & 6.1 \\
\hline & Standard deviation & 6.7 \\
\hline
\end{tabular}

mean length of working in dementia nursing wards 4.4 years $(\mathrm{SD}=2.5)$, and 3 were certified nurses in dementia nursing and 1 certified nurse specialist in gerontological nursing.

\subsection{Condition to Decide on Admission to Dementia Nursing Wards in Psychiatric Hospitals}

From the interview data, 1783 statements were obtained. Excluding 562 that were not relevant to the "statements related to the admission of people with dementia to dementia nursing wards", we included the remaining 1221 statements in the analysis. Table 2 shows the results of the analysis of these, which yielded 4 categories, and 44 codes. In the following, the categories will be indicated with square brackets ([ ]) and codes with angular brackets $(<>)$, and the number of the categories and codes are indicated in parentheses $(())$.

The categories are [Showing a state where self-control is difficult (8)], [Insufficient support provided (10)], [Appearance of symptoms that make daily life difficult (20)], and [Appearance of physical symptoms that require treatment (6)]. [Showing a state where self-control is difficult (8)] is comprised of states 
Table 2. Condition to decide on admission to dementia nursing wards in psychiatric hospitals.

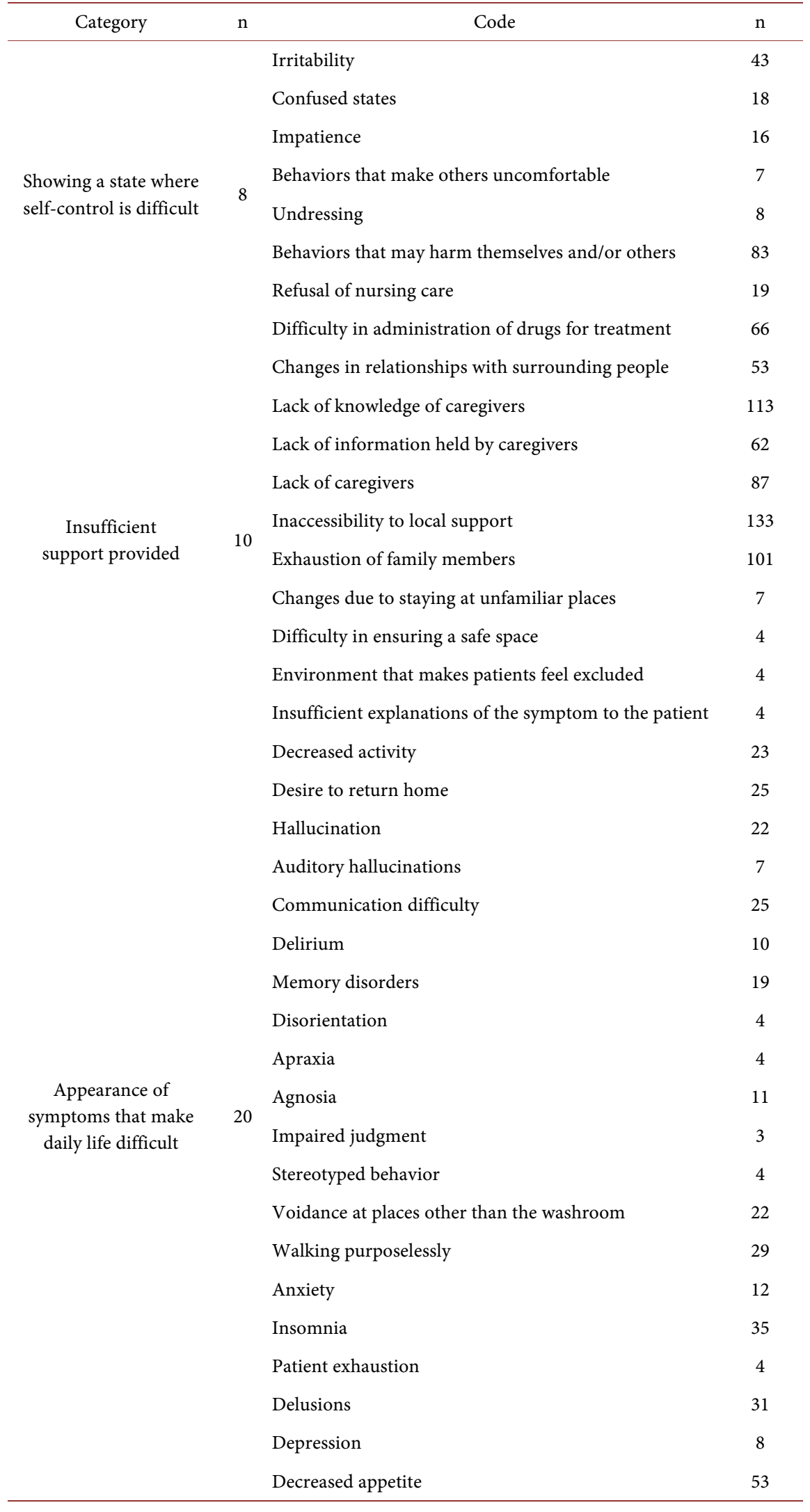




\begin{tabular}{clc}
\hline & Pain & 1 \\
& Itching & 3 \\
$\begin{array}{c}\text { Appearance of physical } \\
\text { symptoms that require } \\
\text { treatment }\end{array}$ & Dehydration & 12 \\
& Undernutrition & 5 \\
& Internal diseases & 16 \\
& Constipation & 4 \\
\hline
\end{tabular}

where people with dementia cannot control their behavior by themselves, and this category includes <irritability (43) $>$, <confused states (18) $>$, <impatience (16) $>$, <behaviors that make others uncomfortable (7) $>$, <undressing $(8)>$, $<$ behaviors that may harm themselves and/or others (83) $>$, $<$ refusal of nursing care (19) $>$, and $<$ difficulty in administration of drugs for treatment (66) $>$. $<$ Irritability (43) > was derived from statements such as "I feel that it is difficult for patients who have short tempers (to return to their former living environment)". $<$ Undressing (8)> was derived from statements such as "Patients who can move their limbs often take off their diapers, so it is difficult for me to take care of such patients". <Behaviors that may harm themselves and/or others (83)> was from statements such as "the patients (with dementia) were hospitalized due to violence against other family members". <Difficulty in administration of drugs for treatment (66)> from "Adjusting drugs to be administered was difficult and it took about a year to establish the criteria for administering a drug".

[Insufficient support provided (10)] is comprised of statements where the conditions for dealing with the patients with dementia were not sufficiently prepared, and this category includes <changes in relationships with surrounding people (53)>, <lack of knowledge of caregivers (113)>, <lack of information held by caregivers $(62)\rangle$, <lack of caregivers (87) >, <inaccessibility to local support (133) >, <exhaustion of family members (101) >, <changes due to staying at unfamiliar places (7) $>$, < difficulty in ensuring a safe space $(4)>$, <environment that makes patients feel excluded (4)>, and <insufficient explanations of the symptom to the patient $(4)>$. $<$ Changes in relationships with surrounding people (53)> was derived from statements such as "I cannot accept the symptoms of the dementia, although I know it is because of the illness". <Lack of knowledge of caregivers (113)> was from statements such as "Patient families had been scolding and violent to the patients before admission because they did not know how to deal with patients with dementia". <Lack of information held by caregivers (62) > was derived from statements such as "Because the facility did not know how to respond to the patient according to the individuality of the patient, symptoms appeared and resulted in the hospitalization". <Inaccessibility to local support (133)> was from statements such as "If caregivers do not know available services, they will become exhausted and patients will be hospitalized due to difficulty in living daily lives". <Insufficient explanations of the symptom to the patient (4)> was from statements such as "Because (the patient) did not know what 
to do when hallucinations appeared, the symptoms worsened and the patient was hospitalized".

[Appearance of symptoms that make daily life difficult (20)] is comprised of states where there were symptoms and these symptoms interfered with daily life, and this category includes $<$ decreased activity $(23)>$, $<$ desire to return home (25) $>$, <hallucination (22) $>$, < auditory hallucinations (7) $>$, < communication difficulty (25) $>$, $\langle$ delirium (10) $\rangle$, $\langle$ memory disorders (19) $\rangle$, $\langle$ disorientation (4) $>$, $<$ apraxia (4) >, <agnosia (11) >, <impaired judgment (3) >, <stereotyped behavior (4) $>$, $\langle$ voidance at places other than the washroom (22) $\rangle$, $\langle$ walking purposelessly (29) $>$, < anxiety (12) >, <insomnia (35) $>$, <patient exhaustion (4) $>$, < delusions $(31)>,\langle$ depression $(8)>$, and $<$ decreased appetite (53) $>$. $<$ Memory disorders (19) $>$ was derived from statements such as "The patient bought a lot of the same items because they forgot previous buying due to memory disorders". $<$ Voidance at other places than the washroom (22)> was from statements such as "People with dementia urinated and defecated on the floors at home or in the garden outside their home". <Patient exhaustion (4)> was from statements such as "I think that the patient with dementia feels afflicted because he also looks distressed without knowing what to do". <Decreased appetite (53) > was from statements such as "The patient was hospitalized at first for the purpose of improving his dietary intake”. <walking purposelessly (29)> was from statements such as "The reason why the patient was hospitalized was that the patient gets lost (when going out alone) and is taken into custody".

[Appearance of physical symptoms that require treatment (6)] is comprised of states where behavioral and psychological symptoms of dementia (BPSD) are triggered by the appearance of symptoms that require treatment, and this category includes $\langle$ pain $(1)>$, <itching (3) >, < dehydration (12) $>$, <undernutrition (5) $>$, <internal diseases (16) $>$, and $<$ constipation (4) $>$. $<$ Dehydration (12) $>$ was derived from statements such as "There were some cases where patients were hospitalized when BPSD worsened due to dehydration". <Constipation (4)> was from statements such as "Constipation could be the underlying cause of various problems (BPSD)".

\section{Discussion}

Mitsubayashi reported that the incidence of the BPSD at the time of admission to a dementia-specialized unit of a psychiatric hospital for the elderly with Alzheimer's disease is about 90\% [12]. Further, Mitsubayashi stated that the appearance of BPSD increases the burden on caregivers and family members living together with the BPSD patient, and this makes the living at home and at a care facility difficult, leading to inpatient treatment at a psychiatric hospital [8]. The present study identified four categories: [Appearance of a state where self-control is difficult (8)] expresses a state where patients cannot control themselves, and the remaining three categories [Insufficiency of support required (10)], [Appearance of symptoms that make daily life difficult (20)], and [Appearance of 
physical symptoms that require treatment (7)] express the states that may trigger the appearance of BPSD. These show that the appearance of BPSD is a criterion for determining hospitalization. Based on this, we will discuss each category separately below.

\subsection{Hospitalization Due to the [Appearance of a State Where Self-Control Is Difficult (8)]}

[Appearance of a state where self-control is difficult (8)] includes <irritability (43) $>$ and $<$ refusal of nursing care (19) $>$. A previous study stated that when a person with dementia experiences a provocative stimulus from the outside, the feeling of anger is more likely to develop because the person is afflicted by auditory and visual impairment and the cognition (stimulus) appears as distorted by delusions of persecution [13]. For the patient it is a very difficult situation because the patient is sensitive to external stimuli. However, those around the person think that such states will lead to trouble in the nursing care, and this idea may increase the risk of the person being admitted to the dementia nursing ward of a psychiatric hospital.

The first option for treating dementia is caring, and the second option is drug treatment [14]. For BPSD, drug treatment often comes before nursing and other care because by the time of the consultation it has already become difficult for the family to deal with the BPSD [15]. Further, there may be some patients who feel distressed due to the BPSD [14]. For these reasons, drug treatment may be selected for people with dementia. However, the incidence of adverse drug events in older people is higher than that in younger people due to changes in pharmacokinetics by aging and due to polypharmaceutical effects due to treatment for multiple diseases [16]. When patients are older people with dementia, it difficult for physicians to identify whether the patients have taken drugs at home because physicians collect information about the BPSD at home or facility from the caregiver rather than directly from the patients. This may explain why it is decided that the patients need to be hospitalized, for patients with < difficulties in administration of drugs for treatment (66)>.

\subsection{Hospitalization Due to [Insufficient Support Provided (10)]}

[Insufficient support provided (10)] includes <lack of knowledge of caregivers (113) > and <lack of information held by caregivers (62)>, showing that caregivers need more information and that information quality is also important. Lack of information provision may lead to the <exhaustion of family members (101)> and $<$ changes in relationships with surrounding people (53) $>$, resulting in the necessity for hospitalization. In addition, as stated in <changes in the relationships with surrounding people (53)>, there are cases where a person with dementia was hospitalized because of violent behaviors towards family members. As described earlier, people with dementia are sensitive to external stimuli [17], violent behaviors may be caused by the symptoms of dementia. However, because family caregivers cannot accept the violent behaviors as a symptom of de- 
mentia, the relationship between the two parties deteriorates, and it will be difficult to accept the necessary support in a familiar environment. As a result, the family thinks it necessary to change the environment, which then leads to hospitalization.

Suzuki described the nurse perception of older people with dementia, stating that nurses often think of older people with dementia as "people who cannot understand anything", "people who do not understand anything", and "people who do not think about anything and cannot complain" [17], and labeling of the person as a person who does not understand because of dementia. This may explain the reason why a code <insufficient explanations of the symptom to the patient (4) $>$ is included in [Insufficient support provided (10)].

\subsection{Hospitalization Due to [Appearance of Symptoms That Make Daily Life Difficult (20)]}

[Appearance of symptoms that make daily life difficult (20)] shows that patients are hospitalized due to <memory disorders (19)>, including the state where symptoms have appeared and the state where symptoms have been obstacles in daily life, such as purchasing many of the same items. Further, codes such as $<$ appetite loss (53)> may lead to the risk of < undernutrition (5)> are included in [Appearance of physical symptoms that require treatment (6)] if the symptoms persist. In addition, <walking purposelessly (29)> not only causes the requirement of long-term care, but also increases the risk of falls. This symptom makes it difficult to continue nursing care, and may increase the possibility of hospitalization of people with dementia if their physical symptoms worsen.

\subsection{Hospitalization Due to [Appearance of Physical Symptoms That Require Treatment (6)]}

As the statements related to <dehydration (12) > and <constipation (4) $>$ in [Appearance of physical symptoms that require treatment (6)] show, these symptoms trigger BPSD. Suzuki [18] reported that older people with severe dementia develop symptoms such as irritation and excitement due to confusion, which makes nursing care more difficult. Specifically in cases where BPSD symptoms have appeared, symptoms are more likely to worsen because these complaints are difficult to identify, and it will be difficult to provide care in general wards. This may explain the reason why the hospitalization in dementia nursing wards in psychiatric hospitals is selected.

\subsection{Nursing Implications and Suggestions}

\subsubsection{Support to Avoid Hospitalization}

The results of the present study show that it is possible to avoid hospitalizing people with dementia in a dementia nursing ward of a psychiatric hospital if nurses have proper knowledge about dementia, understand potential states properly, and prepare an environment where patients can receive the necessary support. Further, patients with dementia will be able to live in familiar places by 
preventing the appearance of BPSD, which may reduce the need for hospitalization. These suggests the necessity for nurses to assist patients with dementia to avoid being hospitalized by observing the patients so that the states described in the codes do not appear, and by preparing an accommodating environment. Clinical symptoms shown in patients with dementia with Lewy bodies are mainly related to cognitive dysfunction, which often begins with memory disorders, but compared to Alzheimer dementia, the severity of memory disorders is mild in the early stages, and the main symptoms include executive function disorders, deterioration of problem solving ability, constructional disorders, visual spatial disabilities, and attention disturbance [15]. Further, hallucination is a characteristic clinical symptom, and patients may also experience, a state where people feel that there is something that is not actually visible [15]. In other words, depending on the severity and type of dementia, patients can alleviate confusion by understanding what is happening to themselves. This suggests that explaining the symptoms according to the capacity of the patients may lead to avoiding patient confusion, in effect providing assistance to avoid hospitalization.

\subsubsection{Appropriate Timing of Hospitalization}

Nakane et al. reported that patients suffering from schizophrenia and depression in Japan had stronger negative attitudes than those in Australia [19]. This suggests that people in Japan may have more negative impressions towards mental illnesses, and that they may hesitate to be admitted to the dementia nursing ward of a psychiatric hospital. As a result, people with dementia will not be hospitalized with appropriate timing, and will be at a higher risk of worsening their symptoms. The codes identified in the present study show the states to decide on hospitalization. By creating a checklist based on these findings, we expect that medical professionals can use these to determine the appropriate timing of hospitalization.

\section{Conclusions}

The findings of the study are summarized as follows:

- Analysis yielded 4 categories: [Showing a state where self-control is difficult (8)], [Insufficient support provided (10)], [Appearance of symptoms that make daily life difficult (20)], and [Appearance of physical symptoms that require treatment (7)].

- The 4 categories show the states where BPSD has appeared and the conditions that trigger the appearance of BPSD. The appearance of BPSD was the criterion for determining hospitalization.

- By providing support to prevent the states described in the 44 codes, nurses may help people with dementia avoid being hospitalized in dementia nursing wards in psychiatric hospitals.

- By creating a checklist based on the 44 codes, nurses can determine appropriate timings for hospitalization in dementia nursing wards in psychiatric hospitals. 


\section{Acknowledgements}

We wish to express our gratitude to the participants and staff of all the participating hospitals for their kind cooperation in conducting this research. This research is funded by research funds allocated to the researcher (head author) by Hokkaido Bunkyo University, and the funds are properly managed and disbursed through the Accounting Division of Hokkaido Bunkyo University. The authors declare that the research was conducted in the absence of any commercial or financial relationships that could be construed as a potential conflict of interest.

\section{Conflicts of Interest}

The authors declare no conflicts of interest regarding the publication of this paper.

\section{References}

[1] Health, Labour and Welfare Statistics Association (2020) Population Survey by Age, Index of National Health Trends/Welfare. Special Edition, Health, Labour and Welfare Statistics Association Publishing, Tokyo, 47.

[2] Ministry of Health, Labour and Welfare (2018) Outline for Promoting Dementia Measures. https://www.mhlw.go.jp/content/000522832.pdf

[3] Cabinet Office (2016) White Paper on Older People. https://www8.cao.go.jp/kourei/whitepaper/w-2017/html/gaiyou/s1 2 3.html

[4] Asada, T. (2012) Dementia Care in Psychiatric Wards: Aiming to Shorten Hospital Stays, Dementia, Incidence, and Future Psychiatric Resources. Japanese Journal of Geriatric Psychiatry, 23, 535-543. (in Japanese)

[5] Katamaru, M., Miyajima, N. and Murakami, S. (2010) An Investigate of Sleep and Behavioral and Psychological Symptoms of Dementia (BPSD) in Elderly Patients with Dementia in Mental Hospitals, and the Examination of Nursing Intervention to BPSD: Comparison between Dementia of Alzheimer Type and Vascular Dementia and Dementia with Lewy Bodies. Japanese Journal of Geriatric Psychiatry, 21 445-455. (in Japanese)

[6] Kawamura, N. (2014) Experimental Animal-Assisted Therapy for Elderly Dementia People Based on an Analysis of Utterances toward Animals. Journal of Animal-Assisted Education and Therapy, 5, 1-12.

[7] Tanaka, Y. and Saeki, H. (2017) The Structure of Decision-Making about the Discharge of Persons with Early Onset Dementia from a Psychiatric Facility among Family Caregivers. Journal of Japan Academy of Community Health Nursing, 20, 46-54. (in Japanese)

[8] Mitsubayashi, S. and Yamada, K. (2013) Six-Month of Experience with Falls of Elderly Patients in a Ward Specializing in Dementia at a Psychiatric Hospital and the Relationship with Behavioral and Psychological Symptoms in Dementia. Japanese Journal of Nursing Research, 36, 49-55.

[9] Kobayashi, O., Ito, H., Iwama, H., Iwanari, H. and Fujita, J. (2009) Use of Pro Re nata Medication in a Public Psychiatric Hospital. Clinical Psychiatry, 51, 567-577.

[10] Ibe, T. (2005) Benner Nursing Theory: New Interpretations-From Beginner to Fully Mastering. Igaku-Shoin Ltd., Tokyo. 
[11] Berelson, B. (1957) Content Analysis (Inaba, M., et al., Trans.). Misuzu Shobo, Tokyo.

[12] Mitsubayashi, S. (2015) Survey of the Incidence of Behavioral and Psychological Symptoms of Dementia in a Psychiatric Hospital for Elderly with Alzheimer Dementia. Kyoto Nursing Journal, 1, 79-85.

[13] Fujisaki, A. (2016) Chapter 4-6, Assessment and Care for People with BPSD: Treatment Refusal. In: Dementia Care Guidebook of Japanese Nursing Association, Shorinsha, Tokyo, 19-23.

https://www.nurse.or.jp/nursing/practice/ninchisyo/pdf/careguide en.pdf

[14] Nakajima, K. (2017) Nursing for People with Dementia. 3rd Edition, Ishiyaku Publishers, Inc., Tokyo, 83.

[15] Sumi, Y. (2016) Chapter 1-6, Pharmacologic Treatment. In: Dementia Care Guidebook, Japanese Nursing Association, Shorinsha, Tokyo, 36.

[16] Nagase, A. and Kitagawa, K. (Ed.) (2018) Adverse Drugs Events Characteristic to the Elderly, Nursing for Elderly Patients Undergoing Drug Treatment, Chapter 7 Nursing for the Elderly in Need of Treatment. In: Nagase, A. and Kitagawa, K., Eds., Systematic Nursing Course Specialized Field II: Geriatric Nursing, Igaku-Shoin, Tokyo, 323.

[17] Suzuki, M. (2016) Care for the Elderly with Dementia Who Undergo Treatment at Acute Care Hospitals from a Person-Centered Perspective: From Admission to Community Participation after the Discharge. Japanese Nursing Association Publishing Company, Tokyo, 4.

[18] Suzuki, M. (2016) Step-Up Dementia Nursing in Acute Care Hospitals Based on the Proficiency Stage of Practical Nursing Skills. Japanese Nursing Association Publishing Company, Tokyo, 18.

[19] Nakane, Y., Yoshioka, K. and Nakane, H. (2010) Aiming to Instill a Barrier-Free Mind. In: Imura, H., Ed., Depression and Schizophrenia for Japanese. Keiso Shobo, Tokyo, 76. 